\title{
LA GESTIÓN INTEGRAL DE LOS RESIDUOS SÓLIDOS URBANOS EN JUÁREZ: LECCIONES INNOVADORAS PARA OTROS MUNICIPIOS
}

\author{
Ismael Couto Benítez \\ ismaelcouto@gmail.com \\ H. Ayuntamiento de Puebla
}

Alberto Hernández ahdez@colef.mx

Cecilia Sarabia csarabia@colef.mx

El Colegio de la Frontera Norte

\section{RESUMEN}

El arribo de la iniciativa privada a la gestión integral de residuos sólidos urbanos en el municipio de Ciudad Juárez promovió un nuevo mecanismo institucional para operar un servicio público que ha cumplido las expectativas de mejora y eficiencia establecidos por los indicadores de gerenciamiento para el servicio de limpieza pública, además de solventar el problema de la falta de presupuesto para el mantenimiento y renovación del equipo de recolección de basura.

Este documento aborda, a partir de una revisión histórica de datos y entrevistas con los directivos de la empresa PASA y los funcionarios del gobierno municipal de Juárez, el modelo en que se gestó y la forma en que opera el servicio de limpia en uno de los cinco municipios más poblados de México. A partir de esta experiencia, se asumen nuevos retos para la administración de los servicios públicos municipales a partir de prácticas de negociación, calidad en el servicio, rendición de cuentas y control gubernamental.

Palabras clave: gestión pública, residuos sólidos, servicios públicos, manejo de conflictos. 


\section{ABSTRACT}

The integration of the private sector into comprehensive management of urban solid waste in the municipality of Ciudad Juárez shows a new institutional mechanism to operate a public service that has fulfilled the improvement and efficiency expectations established by managerial indicators for public sanitation services, in addition to overcoming the lack of budget provision for maintenance and renewal of waste collection equipment.

By means of a historical revision of data as well as interviews with both executive directors of a garbage-collecting firm called PASA and officials of the municipal government of Ciudad Juárez, this document approaches the waste collection model that was created as well as the way in which the garbage collection service operates in one of the five most populated municipalities in Mexico. Based on this experience, the management of municipal public services assumed new challenges by using negotiation practices, quality of service, financial accountability and government control.

Key Words: public management, solid waste, public services, conflict management. 


\section{ELCONTEXTO MUNICIPAL}

Ciudad Juárez es uno de los cinco municipios más poblados de México. Esta ciudad fronteriza del estado de Chihuahua se ha caracterizado por la gran cantidad de empresas maquiladoras y la consecuente oferta de mano de obra. Cuenta con una alta concentración de población inmigrante y fue el primer municipio del país en crear un Instituto Municipal de Planeación e Investigación. Es pionero en procesos de alternancia política. Desde hace una década imperan en este espacio geográfico condiciones extremas de inseguridad como consecuencia del crimen organizado y el narcotráfico. Ello le ha generado una imagen negativa a nivel nacional y mundial.

Por su concentración de población y sus indicadores económicos, el municipio de Juárez se ha perfilado como uno de los centros urbanos más importantes del estado de Chihuahua. Actualmente, Juárez cuenta con la mayor población de los municipios del estado, 1,332,131 habitantes (INEGI 2010). En el lapso de 1950 a 2000, ${ }^{1}$ presentó tasas de crecimiento que oscilaron entre 4.35 y 8.85. Sin embargo, en la última década tuvo una tasa de crecimiento promedio anual de sólo 0.86 , lo cual se ha relacionado con varias hipótesis, como la crisis económica de Estados Unidos y su efecto en el sector industrial local o el retorno de inmigrantes, principalmente veracruzanos, a sus lugares de origen a causa de la inseguridad.

En relación a este último aspecto, los datos de migración indican que, en la actualidad, Juárez es el municipio del estado que cuenta con el mayor porcentaje de habitantes nacidos en otra entidad o país, un 31.9\%. La revisión histórica de este rubro indica que desde 1950 a la fecha, el promedio de la población que habita en la ciudad y que ha nacido en otra entidad federativa es del 30\%, manteniendo una oscilación entre el $23 \%$ y el 38\%. El repunte en el flujo migratorio aflora en la década de los sesenta, coincidiendo precisamente con el impulso de la industria maquiladora, cuando el total de habitantes nacidos en otra entidad federativa alcanzó el $42 \%$. 
Este fenómeno migratorio no puede ser entendido por completo sin tomar en cuenta la importancia de la industria maquiladora en la ciudad, por la creación de empleo y el aporte de divisas a la economía. La dinámica laboral de la industria maquiladora requirió un alto número de trabajadores, sobre todo en las décadas de 1980 y 1990 cuando, según los datos de las estadísticas de la industria maquiladora de exportación, el incremento de establecimientos y el empleo en el sector aumentaron en casi un 100\% (INEGI 1986), lo que desencadenó un crecimiento de la mancha urbana incrementando la demanda de urbanización y la necesidad de dotación de servicios de toda índole.

Resulta difícil precisar datos sobre la generación, recolección, transporte y disposición final de los residuos de la industria maquiladora en virtud de que corresponde a varias instancias de gobierno la actuación sobre cada etapa. El registro como empresas generadoras de residuos y el padrón de recolectores es asunto del gobierno del estado, ${ }^{2}$ mientras que el manejo de residuos peligrosos corresponde a la Secretaría de Medio Ambiente y Recursos Naturales (SEMARNAT). En el municipio, concierne a la Dirección de Ecología y Protección Civil integrar el registro de los residuos recolectados de comercios, servicios e industria maquiladora sin hacer ninguna distinción por sector. Sin embargo, por la cantidad de industrias maquiladoras en la localidad, se puede estimar que la mayoría de los datos sobre la recolección y el transporte de los residuos no peligrosos que se registran en este ámbito de gobierno corresponden a este sector.

De acuerdo a los registros semestrales de las 111 empresas y personas autorizadas para la recolección de residuos sólidos no peligrosos recolectores autorizados, ${ }^{3}$ el siguiente cuadro condensa la información estimada sobre la recolección y disposición de residuos no peligrosos durante el año 2010 y el periodo de enero a julio del 2011. 
Cuadro 1. Residuos recolectados en comercios, servicios e industrias maquiladoras y su destino final

\begin{tabular}{|c|c|c|c|}
\hline \multirow{2}{*}{ Residuos recolectados } & \multicolumn{2}{|c|}{ Toneladas } & \multirow{2}{*}{ Destino final } \\
\hline & 2010 & 2011 & \\
\hline Alfombras & 217.93 & 230.34 & Centros de acopio/tratamiento y recicladoras \\
\hline Aserrín de carne y hueso & 521.66 & 90.57 & Centros de acopio/tratamiento y recicladoras \\
\hline Aserrín de madera & 10.00 & 2.40 & Relleno sanitario y recicladoras \\
\hline Basura industrial-Srap & $12,186.02$ & $1,211.95$ & Retorno a EEUU \\
\hline Cartón & $139,089.15$ & $59,016.21$ & Centros de acopio/tratamiento y recicladoras \\
\hline Desperdicio de pasta & 532.26 & 181.00 & Retorno a EEUU y relleno sanitario \\
\hline Escombros de construcción & $4,101.43$ & $1,121.34$ & Relleno sanitario \\
\hline Esponjas & 20.15 & 25.11 & Centros de acopio/tratamiento y recicladoras \\
\hline Hueso animal & $1,789.92$ & 885.06 & Centros de acopio/tratamiento y recicladoras \\
\hline Leña & 933.60 & & $\begin{array}{l}\text { Relleno sanitario y recicladoras (un porcentaje } \\
\text { se regala para leña) }\end{array}$ \\
\hline Metales ferrosos & $73,977.01$ & $38,894.31$ & Centros de acopio y recicladoras \\
\hline Metales no ferrosos & $2,038.32$ & & Centros de acopio y recicladoras \\
\hline Nata de pintura & 1.00 & & Relleno sanitario \\
\hline Papel & $14,256.20$ & $3,588.66$ & Centros de acopio/tratamiento y recicladoras \\
\hline Piel & 2.15 & 9.35 & Retorno a EEUU y relleno sanitario \\
\hline Plásticos en general & $17,891.31$ & $7,970.14$ & Centros de acopio/tratamiento y recicladoras \\
\hline Poliestireno (nieve seca) & 31.17 & 149.63 & Centros de acopio/tratamiento y recicladoras \\
\hline Residuos no clasificados & $2,001.37$ & $4,324.48$ & $\begin{array}{l}\text { Relleno sanitario, centros } \\
\text { acopio/tratamiento y recicladoras }\end{array}$ \\
\hline Residuos sólidos urbanos (basura) & $75,397.91$ & $22,823.13$ & Relleno sanitario \\
\hline Sebo animal & $1,882.59$ & $1,975.75$ & Centros de acopio/tratamiento y recicladoras \\
\hline Telas & 317.76 & 394.24 & Centros de acopio/tratamiento y recicladoras \\
\hline Vidrio & 31.55 & 34.37 & Retorno a EEUU y relleno sanitario \\
\hline Vinil & $1,219.89$ & $1,539.92$ & Retorno a EEUU y relleno sanitario \\
\hline \multicolumn{4}{|c|}{ Residuos recolectados por unidad } \\
\hline Cartuchos de toner & 31,627 & 2,796 & Centros de acopio y recicladoras \\
\hline $\begin{array}{l}\text { Orgánicos alimenticios (Escamocha) } \\
\text { (tambos de } 200 \mathrm{lt})\end{array}$ & 151,425 & 140,999 & Centros de acopio y relleno sanitario \\
\hline Tambos de cartón & 3,258 & 378 & Centros de acopio/tratamiento y recicladoras \\
\hline Tarimas de madera & $2,285,663$ & $1,084,944$ & Centros de acopio/tratamiento y recicladoras \\
\hline \multicolumn{4}{|c|}{ Residuos recolectados por litros } \\
\hline $\begin{array}{l}\text { Aceites y/o grasas de origen animal } \\
\text { y/o vegetal }\end{array}$ & $536,532.00$ & $4,324.48$ & Centros de acopio/tratamiento \\
\hline Trampas de grasas & $6,799,181.80$ & $2,911.41$ & Centros de tratamiento \\
\hline
\end{tabular}

Fuente: Dirección General de Ecología y Protección Civil, Gobierno Municipal 2010-2013.

La inmigración generada por la oferta de mano de obra en el sector industrial propició el crecimiento acelerado, desordenado e incontrolable de la ciudad a partir de múltiples asentamientos irregulares que, por invasión o autoinvasión, aparecían de manera espontánea; este aumento demográfico y la expansión territorial provocaron grandes rezagos urbanos, sobre todo en cuanto a pavimentación y servicios públicos (Fuentes 2009), 
propiciando el florecimiento de zonas marginadas.

En la zona poniente fue donde más proliferaron este tipo de asentamientos, que se establecían con el mínimo de recursos para sobrevivir, sin la dotación de ningún servicio y con escasas posibilidades para conseguirlos ya que la Ley de Desarrollo Urbano del estado de Chihuahua ${ }^{4}$ prohíbe brindar servicios públicos a personas que carecen de personalidad legal o que ocupan un predio que no es de su propiedad.

Aunque la tenencia de la tierra fue un factor fundamental para la dotación de servicios públicos, para la década de 1960 había sólo un 31.8\% de viviendas ocupadas por propietarios $\mathrm{y}$, aunque esto no significa que no estuvieran en proceso de pago, sí es una referencia de la gran cantidad de predios y viviendas sin regularizar que, por ende, carecían de servicios. Sin embargo, en las décadas siguientes la cantidad de propietarios aumentó considerablemente de un $50.8 \%$ en 1970 , a un $57.6 \%$ entre 1970 y 1980 , y por encima del $70 \%$ en las décadas subsiguientes.

En cuanto a las viviendas, los datos más recientes del Censo General de Población y Vivienda 2010 indican que el municipio cuenta con un total de 488,785 viviendas particulares, de las cuales 364,562 están habitadas por un promedio de 3.6 ocupantes. Del total de viviendas, un $22.7 \%$, correspondiente a 111,103 , son viviendas deshabitadas, es decir, nuevas y que alguna vez fueron habitadas; y un 2.7\%, equivalente a 13,120 viviendas, son de uso temporal, siendo el porcentaje total de viviendas deshabitadas y de uso temporal del 25.41\%. Esta situación no es nueva ya que desde el 2005 existían un 17\% de casas abandonadas que posicionaban al municipio en el primer lugar nacional en cuanto a porcentaje de viviendas deshabitadas (Rubio 2011). Siendo el municipio de Juárez una ciudad policéntrica que rompe los esquemas de provisión de servicios públicos, se requiere de planes innovadores para asumir la demanda de servicios públicos exigida por la población. 
Los datos más recientes de dotación de servicios públicos muestran que, a la fecha, el municipio cuenta con un $98.8 \%$ de viviendas particulares que disponen del servicio de energía eléctrica, un $97.6 \%$ que tienen agua entubaba y un $97.9 \%$ que cuentan con drenaje. A partir de estos datos se puede inferir, de acuerdo con la información de la Dirección de Limpia, que la dotación del servicio de recolección domiciliaria de residuos sólidos presenta una cobertura muy amplia o casi completa de la ciudad.

Aunado a todo esto, al analizar el contexto de Juárez es de suma importancia considerar que la proximidad y accesibilidad de la población a la vecina ciudad estadounidense de El Paso, Texas, favorece la identificación de las diferencias entre una ciudad y otra en todos los aspectos, a lo que no escapa la urbanización y la dotación de servicios públicos.

Otra característica del contexto de Juárez es el fenómeno de la alternancia política que inició en el gobierno municipal para continuar en el estatal. Al igual que el resto del país, la ciudad estuvo gobernada por el Partido Revolucionario Institucional (PRI) hasta la década de los ochenta. Posteriormente, el Partido Acción Nacional (PAN) estuvo al frente del gobierno municipal en el periodo de 1986 a 2004, pero los resultados electorales en los últimos periodos muestran de manera muy clara una tendencia que favorece nuevamente al PRI como primera fuerza política, dándose así la doble alternancia en el gobierno local. Ahora, el PAN es la segunda fuerza, y el Partido de la Revolución Democrática (PRD), que hasta el año 2007 fue la tercera, ha sido desplazado por el Partido Verde Ecologista de México.

En el contexto de doble alternancia, PRI-PAN-PRI, el municipio ha experimentado en las dos últimas décadas distintas formas de oposición y de gobierno dependiendo de la ideología política en el poder. En lo que respecta a la externalización de los servicios públicos, por ejemplo, ambos partidos han estado en contra mientras son oposición y a favor cuando están al frente del gobierno. ${ }^{5}$ 
En 1998, la administración municipal panista generó un nuevo reto al establecer una visión de futuro para el manejo de los residuos sólidos. La realización del Plan Maestro de Residuos Sólidos de Ciudad Juárez, Chihuahua (Cocef 1998), se convirtió en herramienta fundamental para la planeación del manejo y la prestación de este servicio público. Gracias al plan maestro y al financiamiento emprendido por parte del Banco para América del Norte (BDAN), se emprendieron acciones encaminadas a mejorar el servicio de recolección de basura. Entre éstas destacan la descentralización del servicio de limpia, el establecimiento de unidades de transferencia y diversas obras de mejora en rellenos sanitarios.

A finales de 2003 el servicio de limpia en Juárez entró en una fase crítica, lo que llevó a una crisis del modelo de gestión de residuos sólidos. Tras doce años de gobierno en el municipio, el PAN trasladó al nuevo gobierno municipal del PRI un problema de difícil solución. El parque vehicular se encontraba deteriorado, eran elevados los costos de mantenimiento y los vecinos se quejaban de la operación en las estaciones de transferencia y del servicio de recolección, además de que los costos por tonelada recolectada se incrementaron significativamente (Córdova et al. 2006).

Debido a esta situación, las nuevas autoridades municipales se vieron obligadas a buscar nuevos esquemas de mejora para el servicio de limpia. A mediados de 2004 dio inicio el proceso de negociación para concesionar el servicio, a pesar de la oposición del sindicato involucrado y de la politización del tema. El principal argumento para lograr que se aprobara la concesión fue la millonaria inversión que sería necesaria para mantener el sistema de recolección y que, de concesionar el servicio, la ciudad pagaría anualmente la cantidad de 130 millones de pesos (mdp) y se ahorraría 13 mdp por la diferencia de la erogación de ese momento, que era de $143 \mathrm{mdp}$.

En México, el incremento en el volumen de residuos sólidos urbanos ha hecho cada vez más difícil su recolección por el costo económico que representa. Otro factor crítico es la 
vida útil de los rellenos sanitarios y el grave rezago para el control de los tiraderos municipales a cielo abierto. La mayor parte de los municipios del país no ha podido dar cumplimiento a lo establecido en la Ley General de Residuos Sólidos, aprobada en 2003, por lo que se calcula que un tercio de los municipios vierten basura en tiraderos a cielo abierto (Santos 2007: 165).

En el caso de Juárez, el manejo de residuos sólidos urbanos representa una experiencia innovadora, más allá de los municipios fronterizos. Aunque en el país existen diferentes experiencias de este tipo, su gran debilidad ha sido la falta de continuidad. La implementación en Juárez de un programa integral de manejo de residuos sólidos merece ser examinada dada su continuidad a lo largo de tres administraciones municipales y por la amplia cobertura de servicios que cubre.

\section{FORMACIÓN DE NUEVAS PRIORIDADES Y PROGRAMAS DE ACCIÓN PÚBLICA}

Tradicionalmente, la administración del municipio de Juárez ha sido dirigida por un núcleo de empleados públicos o burócratas, ${ }^{6}$ sean expertos o personas de confianza política, encargados de tomar decisiones en las diversas áreas de competencia y cuya actividad se ha centrado en la cobertura y la agilización de trámites administrativos propios. Esta tendencia obedece a la idea de un Estado interventor y proteccionista que concibe la tarea de administrar como exclusiva del gobierno.

Sin embargo, hoy por hoy, el gobierno municipal ha tenido que aplicar otros mecanismos que le permitan asegurar la viabilidad económica, realizar sus funciones básicas con eficacia y eficiencia, sin perder la centralidad en el ciudadano o usuario-cliente y rebasando los límites de su estructura (institucional y humana), organización, programas y propuestas (Aguilar 2006). $^{7}$

En el plano municipal emergen nuevas formas organizacionales donde el gobierno 
establece contratos con terceras personas, sean asociaciones o empresas lucrativas o no lucrativas, para aplicar las políticas públicas y con la idea de reducir los costos directos para el ciudadano contribuyente. La interconexión de este ámbito del Estado y la sociedad es una tendencia que ha transformado la vida pública, donde el primero deja de ser un monopolio para incluir a nuevos actores en la solución de asuntos públicos. Este asunto no es tema nuevo ${ }^{8}$ pero sí resulta innovador en el ámbito del municipio de Juárez y sobre todo en la gestión de un servicio público por la iniciativa privada.

La gestión de los servicios públicos es muy significativa debido a que su adecuada prestación se traduce en mejor calidad de vida para la población, razón por la cual se convierte en uno de los principales indicadores de evaluación del buen funcionamiento de los gobiernos locales (Del Pino 2004). Por eso, conforme ha pasado el tiempo, la organización pública ha desarrollado su capacidad de producción y manejo de estrategias para la gestión operativa de los servicios públicos tales como el mejoramiento, la manipulación de los flujos de entrada, la ampliación de los horarios de atención, el incremento de las capacidades, entre otras.

Los servicios públicos, además de ser un espacio de proximidad entre el gobierno y la sociedad y un indicativo para la evaluación de los gobernantes, son también un espacio para la solución de un problema público que permite articular a varios actores de la sociedad para la optimización de los recursos y mejorar la calidad de vida. Es por esto que prolifera un nuevo enfoque de la gestión pública bajo la idea de eficiencia a bajo costo, sin descartar la posibilidad de que, si la organización pública no está preparada para suministrar a la ciudadanía determinados servicios cumpliendo los niveles de calidad, ${ }^{9}$ la prestación de los mismos se pueden traspasar a las organizaciones privadas que cuentan con mejor preparación y experiencia para atenderlos.

La esencia de un servicio público estriba en la forma de proveer satisfactores para las necesidades de los gobernados y en la característica de ser prestados por el gobierno y ser 
declarados como tal. En México, la Constitución Política de los Estados Unidos Mexicanos (CPEUM 2005) ${ }^{10}$ establece en el artículo 115 las funciones, las formas de producción, el manejo y la lista mínima de los servicios públicos municipales, y asegura su prestación regular, continua, igualitaria y general, al mismo tiempo que los protege del libre mercado y sus fluctuaciones (Santiago 2009).

Las formas de producir y manejar los servicios en los municipios pueden ser: directa, por concesión o por convenio con el Estado (García del Castillo 1998). En la primer forma es el órgano público responsable quien asume la gestión integral del servicio; en la segunda, el órgano público responsable otorga contratos o subcontrata a empresas privadas para la prestación; y en la tercera, existen mecanismos de transferencia de funciones de un ámbito a otro que regulan la prestación de manera ininterrumpida del servicio.

En la concesión, el poder público siempre mantiene la titularidad en la prestación de la actividad, pero la gestión es prestada a través de los particulares como producto de un contrato administrativo y se mantiene el régimen jurídico vigente que garantiza las condiciones de continuidad, regularidad, uniformidad, igualdad, obligatoriedad y adaptación a las nuevas circunstancias con respecto al servicio público (HernándezMendible 1999).

En este sentido, es importante distinguir la concesión de la privatización como una forma que corresponde a la externalización ${ }^{11}$ de servicios que en sentido estricto configura el modelo de gestión relacional donde «la Administración dirige y controla y las organizaciones privadas gestionan los servicios públicos» (Ramió 2002: 150). Este modelo llevado al extremo, es decir, con la participación indirecta y limitada del Estado en la prestación de los servicios, es el fenómeno del Estado hueco, donde se cede a terceras partes la gestión, financiación e incluso la elegibilidad de los servicios (Milward et al. 1998). 
En el caso particular de Ciudad Juárez, el municipio, en pleno ejercicio de sus derechos y obligaciones, consideró que la inadecuada recolección de los desechos sólidos es dañina para el ecosistema y que, debido al alto costo del manejo integral de los residuos, requería incluir a otros actores, en este caso a la iniciativa privada (Código Municipal para el estado de Chihuahua 2004a, 2004b, 2004c).

Al incluir a empresas especializadas que puedan entender la naturaleza del problema y sus posibilidades de operación y manejo, se ha pasado de ser un sistema centralizado a un sistema más democrático que incluye la participación de actores en las tareas institucionales, lo que fomenta las relaciones horizontales en las actividades tendientes a la gestión integral de los residuos sólidos.

La participación del sector privado en el gobierno a través de la prestación de un servicio público es una práctica novedosa en la ciudad y ha requerido la construcción de la normatividad adecuada, ${ }^{12}$ la cual se complementa con la legislación de otros ámbitos de gobierno que cuentan con mayor experiencia y que tienen la posibilidad de intervenir por un lapso mayor de tiempo en los asuntos públicos.

La idea original de esta modificación proviene de un diagnóstico realizado a cada una de las áreas de los servicios públicos municipales (alumbrado, limpia, mantenimiento de vías, control de tráfico, parques y jardines, etcétera). Este diagnóstico fue dirigido en la administración 2004-2007 por el Ing. Héctor Murguía Lardizábal, quien actualmente es el presidente municipal. Aunque anteriormente el servicio de limpia no era malo, sí operaba con un alto costo; se contaba con una flotilla de camiones cuyas unidades de recolección eran obsoletas o de modelos con más de treinta años de antigüedad (los modelos más recientes en ese año eran de 2001) que por supuesto no cumplían con los estándares internacionales para ofrecer el servicio de limpia y cuyo mantenimiento costaba doce millones de pesos al año en promedio. ${ }^{13}$ 
En este tenor, la concesión del servicio se realizó en función de evitar el gasto de la compra de unidades y barredoras nuevas para el servicio de recolección, y por la necesidad de ampliar las rutas del servicio y terminar con el problema de los pepenadores, ${ }^{14}$ es decir, hacer más eficiente ${ }^{15}$ la prestación del servicio operando a un menor costo a partir de un nuevo proyecto de recolección y disposición final de residuos sólidos.

Bajo estos lineamientos y modelo, Promotora Ambiental de la Laguna SA de CV (PASA) realiza, desde el 6 de noviembre de 2006, la recolección de desechos sólidos domiciliarios no peligrosos en Ciudad Juárez después de haber ganado el proceso de licitación nacional.

\section{LA CONCESIÓN DEL SERVICIO DE LIMPIA}

El Código Municipal para el estado de Chihuahua establece las facultades y obligaciones de los ayuntamientos para autorizar la celebración de contratos de obras o servicios públicos municipales o el otorgamiento de concesiones. Por otra parte, el Reglamento Orgánico de la Administración Pública del municipio de Juárez, en el artículo 46, faculta al municipio como responsable y vigilante de las concesiones y del cumplimiento de la normatividad respecto de la gestión de los residuos sólidos. Este mismo reglamento en el artículo 49 establece la responsabilidad total para la Dirección de Limpia por la prestación del servicio independientemente de la forma en que se realice. ${ }^{16}$ Bajo esta normativa se realizó la convocatoria para la concesión de la recolección de residuos sólidos domiciliarios en Ciudad Juárez.

La concesión a la empresa PASA se otorgó «para desarrollar y operar la prestación de servicios públicos de aseo urbano, que comprende el barrido manual, barrido mecánico, recolección, traslado y depósito de los desechos sólidos de carácter domiciliario y edificios públicos municipales y tratamiento y compactación de estos», ${ }^{17}$ bajo un contrato por quince años que inició el 4 de septiembre de 2006 para concluir el 4 de septiembre del año 2021. 
La autorización fue suscrita en la sesión ordinaria número $52 \mathrm{del} \mathrm{H}$. Ayuntamiento que se realizó el 27 de abril de 2006, a nombre de Promotora Ambiental de la Laguna SA de CV, con representación legal del Ing. Isidro Sada García, y cuya denominación comercial lo identifica como servicios de aseo urbano integral.

La concesión del servicio de recolección de residuos sólidos en Juárez se realizó de manera pública con la participación de siete empresas, algunas de origen extranjero.

Las condiciones para otorgar la concesión fueron básicamente cinco:

- Operar con camiones nuevos de modelo 2007.

- Renovación de la flotilla de camiones cada siete años.

- Indemnización a los empleados del municipio y su contratación como personal de la empresa.

- Certificación en la recolección domiciliar y el relleno sanitario como industria limpia por parte de la Procuraduría Federal de Protección al Ambiente (PROFEPA).

- El contrato de concesión incluyó la indemnización y recontratación de los empleados del Departamento de Limpia en la empresa, sin embargo, en esa coyuntura algunos empleados aprovecharon para jubilarse.

Las opiniones y los argumentos incluidos en expedientes y documentos revelan que la aprobación se basó en razones de eficacia, eficiencia y economía para el municipio. 


\section{EL OPERATIVO Y LOS SERVICIOS}

El servicio de PASA abarca la recolección domiciliar, el barrido mecánico y el servicio de aspirado manual en toda la ciudad, además del compromiso conjunto con el municipio para la certificación en la recolección domiciliar y el relleno sanitario como industria limpia por parte de la PROFEPA.

PASA opera con una plantilla de 380 empleados de los cuales casi el $90 \%$ fueron empleados del Departamento de Limpia. Su experiencia previa como operarios y supervisores contribuyó a un mejor funcionamiento del servicio concesionado.

El operativo de recolección se realiza con camiones propiedad de PASA, que suman un total de 107 vehículos compactadores de carga trasera (40 camiones de veinticinco yardas, 56 de veinte yardas y 10 camiones desmontables), con un radio de recolección de 12,200 habitantes por cada vehículo. El mantenimiento, por tanto, queda a cargo de la compañía y se presta cada $3,000 \mathrm{~km} .^{18}$

La cobertura del servicio es total y gratuita, es decir, no existe domicilio por donde no pase el camión recolector y la población del municipio no tiene que pagar nada adicional por el servicio que cubre la administración municipal.

El servicio de recolección de basura recoge en promedio 1,200 toneladas diarias. El lunes y martes los camiones recolectores llevan al relleno sanitario entre 1,500 y 1,600 toneladas; el miércoles, jueves y viernes baja la generación de basura: se recogen de 950 a 1,000 toneladas, y el día sábado se generan de 900 a 1,000 toneladas.

La recolección domiciliar se realiza a partir del establecimiento de rutas, con la propuesta de unificación del servicio tres veces por semana en toda la ciudad, la cual se ha dividido en dos zonas, norte y sur, tomando como referencia la Av. Teófilo Borunda. En la primera 
zona se ofrece el servicio los días lunes, miércoles y viernes, y en la segunda la recolección se realiza martes, jueves y sábado. Adicionalmente, el primer cuadro de la ciudad, llamado Centro Histórico, se cubre los siete días de la semana, pues, por ser una zona muy frecuentada, únicamente ahí se recoge la basura el domingo.

Las zonas periféricas de la ciudad tienen también una cobertura uniforme, pues se llega hasta los límites de la ciudad (del km 27 de terracería, hasta el Ejido El Millón; también se llega a Samalayuca y al Valle de Juárez) en un operativo cuya frecuencia es de dos veces por semana. ${ }^{19}$

El barrido mecánico se realiza con tractobarredoras diariamente por las avenidas primarias y secundarias, cubriendo $360 \mathrm{~km}$.

El barrido manual se implementa en el centro histórico de la ciudad con una cobertura de $26 \mathrm{~km}$ diarios y se complementa en las vías primarias y en los puentes viales cubriendo 64 $\mathrm{km}$ diarios, es decir, se efectúa un barrido total de $90 \mathrm{~km}$ diarios.

El compromiso de cumplir con las leyes, reglamentos y normas ambientales establecidos por la PROFEPA asumido por los establecimientos industriales - que dentro de sus actividades pueden generar emisiones a la atmósfera o dan servicio en materia de residuos peligrosos - ha quedado de manifiesto a partir de la certificación que la dependencia federal emitió a PASA en julio de 2011.

A pesar de este operativo, en la ciudad que, como otras ciudades, tiene una extensa capa urbana sin pavimento, subsisten los tiraderos a cielo abierto. Se estima que existen alrededor de 200 tiraderos clandestinos, aunque no se tiene conocimiento de la localización exacta por su carácter esporádico. Se plantea el supuesto de que la naturaleza de estos tiraderos radica en la falta de acceso por carretera para los camiones recolectores, principalmente en algunas zonas intransitables de la ciudad como Lomas de Poleo, Anapra 
y el km 20 de la salida a Chihuahua. La limpieza de estos tiraderos se realiza conforme se tiene conocimiento de ellos, generalmente por medio de reportes telefónicos al módulo de atención ciudadana o por reportes en los medios de comunicación. En este sentido, el número de quejas ${ }^{20}$ oscila entre 5 y 10 diarias y, por la naturaleza de la supervisión, son atendidas a la brevedad. Es por esta razón que el departamento se maneja con el indicativo de cero quejas.

El municipio realiza un monitoreo constante de la concesionaria en toda la ciudad a través de una sectorización en siete áreas con siete empleados llamados "supervisores de ruta». Estos empleados municipales verifican a su vez el trabajo de los supervisores de PASA, así como el buen funcionamiento de las unidades, de la cobertura y de la frecuencia. Por su parte, la concesionaria supervisa sus propias operaciones por medio de diez supervisores de recolección, uno de barrido manual y uno de barrido mecánico. Este tipo de supervisión obliga a PASA a mantener la calidad del servicio, ya que su eficiencia le ha valido para ampliar la cobertura del servicio e incluso brindar apoyo en programas especiales de la Dirección de Limpia. ${ }^{21}$

\section{CALIDAD Y EFICIENCIA}

El servicio de recolección de basura, antes de la concesión, operaba con mucha inconsistencia; la frecuencia de recolección domiciliaria era de una vez por semana, limitándose sólo en algunas zonas a una frecuencia de dos veces. En la administración 2004-2007 se contaba con 60 camiones recolectores de basura, de los cuales 40 se encontraban en condiciones óptimas para dar el servicio porque eran unidades de 2001; los otros 20 camiones tenían de quince a veinte años de antigüedad y, lógicamente, la cobertura en las rutas muchas veces se hacía hasta altas horas de la noche, es decir, no era un servicio uniforme.

Según establece la Ley General de Residuos, la reconstrucción de las rutas de recolección 
domiciliaria, el incremento en las rutas de barrido y la supervisión del servicio de PASA por parte de la Dirección de Limpia, forman parte de la gestión integral de residuos sólidos. ${ }^{22}$ Este tipo de acciones cumple con las características de continuidad, regularidad, igualdad y obligatoriedad de manera independiente de la administración municipal, ya que forman parte de un contrato.

En el primer año de operaciones de la concesionaria se utilizó un presupuesto de $156 \mathrm{mdp}$ para la gestión integral de los residuos sólidos. Actualmente se opera con $161 \mathrm{mdp}$, lo que significa un incremento de 14 millones, pero el servicio es más eficiente al abarcar la recolección domiciliaria, el barrido mecánico de las vías primarias y secundarias, y programas especiales de barrido manual y semimecánico en el centro histórico de la ciudad.

En cuanto a la operación de la Dirección de Limpia, la planta de trabajadores municipales se redujo un 90\%. En 2006 la nómina ascendía a 650 empleados, mientras que a la fecha suman 68 los empleados en esta Dirección, es decir, se trabaja con mayor eficiencia y ahorro de recursos. Un ejemplo que ilustra la eficiencia conseguida con el modelo de concesión se puede apreciar en el barrido manual que realiza la concesionaria PASA en Ciudad Juárez. Esta actividad se lleva a cabo en el centro histórico con la colaboración de quince empleados que barren $3 \mathrm{~km}$ cada uno por día, mientras que antes de la concesión el municipio barría en total $2.5 \mathrm{~km}$ diarios. ${ }^{23}$

La capacidad de los empleados actuales del municipio permite enfocar recursos a programas especiales de limpia como el destilichadero, programa que recolecta todos aquellos residuos sólidos urbanos «no convencionales» y muy típicos de las ciudades fronterizas: muebles, aparatos electrónicos, electrodomésticos, etc. Otro proyecto específico de la administración municipal es el de recolección de llantas usadas, un problema también grave.

Un aspecto de suma importancia a considerar en la gestión de los residuos sólidos urbanos 
en Juárez es la percepción ciudadana sobre el servicio de la empresa PASA, ya que más allá de los informes y datos que la empresa y el municipio puedan presentar y respaldar, son los beneficiarios directos quienes continuamente evalúan el servicio.

Para analizar este rubro, la Universidad Autónoma de Ciudad Juárez, a través del Instituto de Ciencias Sociales y Administración, realizó en abril de 2011 una evaluación del servicio a usuarios de Promotora Ambiental, $\mathrm{SA}^{24}$ con resultados muy favorables para la empresa. Además de este estudio, un sondeo de opinión de usuarios vía Internet ${ }^{25}$ permitió corroborar la percepción que se tiene del servicio que presta la concesionaria.

En términos generales, en el $100 \%$ de las rutas la opinión de los usuarios coincidió al manifestar satisfacción con el servicio de PASA y en ninguna ruta se reportaron problemas relacionados con la recolección de basura. En el $89.8 \%$ de las rutas, los usuarios reportaron estar satisfechos con el servicio. Al mismo tiempo, la población calificó la frecuencia en la recolección de basura como regular (56.1\%) lo cual, de acuerdo con las opciones de respuesta, equivale a una percepción satisfactoria. En cuanto a los horarios de recolección, se registró un consenso general al manifestar los usuarios que en la mayoría de las rutas $(55.6 \%)$ la basura se recolecta en horario matutino. En relación al servicio del personal de la empresa, en el $97.8 \%$ de las rutas predominan calificaciones de bueno y excelente para los operativos, y de bueno en el $66.3 \%$ de las rutas para la forma en que los choferes conducen las unidades de recolección (UACJ 2011).

Estos datos coinciden con las siguientes opiniones de colonos de ambas rutas del servicio:

«En la colonia es un servicio muy bueno, pasan muy seguido, y por toda la colonia».

Femenino, 23 años, colonia Municipio Libre, Sector 2.

«En general lo califico como bueno. Trabajan incluso días festivos. Hubo un tiempo en que dejaban la mitad de la basura en los tambos pero eso ya mejoro, al parecer cambiaron de 
personal».

Masculino, 28 años, Jardines del Valle, Sector 1.

«[El servicio]... es bueno y puntual, por lo menos en el área donde vivo. Ignoro si dan el mismo servicio en lugares marginados de la ciudad».

Femenino, 58 años, Colonia Villas del Emir, Sector 1.

En lo que se refiere al pago de propinas a los operadores del servicio, en el $97.8 \%$ de las rutas los usuarios indicaron que nunca se les ha solicitado propina (UACJ 2011). En este sentido, el sondeo de opinión permitió corroborar que la práctica de dar dinero a los trabajadores se ha ido diluyendo, pues sólo el 10\% de los usuarios manifestó entregar entre 5 y 10 pesos al realizar la recolección, un 45\% manifestó no dar nada a cambio del servicio y el restante $45 \%$ indicó entregar «a veces» alguna propina, es decir, sin obligación a cambio del servicio.

La evaluación contiene algunas sugerencias a la empresa como: a) corregir el hecho de dejar basura tirada así como el maltrato a los recipientes o botes; b) dar seguimiento a la forma de manejar de los choferes; c) incidir en el posicionamiento de la empresa en la mente del usuario; d) incrementar la capacitación del personal en relación con el manejo de la basura, concientizándolos de la importancia de realizarlo con más detalle y cuidado; y e) continuar con el compromiso de alcanzar buenos resultados, los cuales repercuten en la comunidad entera preservando la imagen de una ciudad limpia y ordenada.

Las sugerencias coinciden con lo expresado por algunos usuarios en el sondeo de opinión:

«...deberían de esperar un poco en cada calle y deberían ser más cuidadosos con los recipientes, los avientan sin precaución».

Masculino, 37 años, Colonia Ruba, Sector 2.

«Excelente, solo hay un pequeño problema, cuando se está recolectando la basura, hay 
veces que se caen bolsas y esas ahí se quedan, no las levantan, pero fuera de eso está muy bien».

Entrevistado, 21 años, Colonia Prados del Real, Sector 2.

«Me parece buen servicio, aunque a veces dejan un poco tirado alrededor de los botes. No son muy puntuales, aunque no sé en qué afecte eso. En general, no tengo quejas».

Masculino, 37 años, Fracc. Arco Iris, Sector 1.

«Considero que es bueno, responsable, pues no importa el día festivo que sea ellos acuden a la recolección, puntuales, el $90 \%$ de las ocasiones pasan a la misma hora, respetuosos, saludan, ¡buenos días!, no te piden cuota, si no sacaste la basura antes, te esperan y ayudan».

Femenino, 35 años, Country Senecú, Sector 1.

Además de los resultados favorables de percepción ciudadana sobre el servicio, recientemente, el 25 de noviembre de 2011, el municipio de Juárez recibió el premio nacional del concurso Limpiemos Nuestro México, promovido por Fundación Azteca de Grupo Salinas, como una de las ciudades más limpias de México. ${ }^{26}$

La cultura del manejo de basura en los domicilios se ha modificado desde que PASA inició la prestación del servicio, por ejemplo, en lo que respecta al uso de los tambos de 200 litros y a la frecuencia, horarios, puntualidad y uniformidad del servicio, aún sin el uso de aviso. ${ }^{27}$ La «cultura del tambo» era un problema grave para los empleados del servicio de limpia debido a la dificultad que representaba el manejo de este tipo de depósitos y su manipulación de forma manual, práctica que poco a poco ha ido cambiando para lograr una mayor eficiencia y optimización de recursos.

Las quejas por el mal servicio se han trocado en reclamos como el de modificar la ubicación de los contenedores de basura. Además de la organización de un operativo acorde a las necesidades, condiciones topográficas y urbanísticas de la ciudad — falta de 
pavimento - y contar con el equipo utilizado, el personal de la empresa PASA ha sido un factor clave para lograr una cultura organizacional que incentiva la eficiencia del servicio.

Otra de las condicionantes de la licitación fue la cobertura del servicio en las zonas de crecimiento de la ciudad. Esta situación se ha modificado debido al abandono de viviendas donde, no obstante, la cobertura continúa con la misma eficiencia y calidad.

\section{ATENCIÓN AL USUARIO}

La principal vía de comunicación con la población es el módulo de atención ciudadana que funciona a través de llamadas telefónicas sobre las fallas del servicio. Para cubrir las quejas que se reciben existen 26 rutas de supervisión con siete cuadrillas de siete trabajadores que atienden los reportes todos los días. Actualmente 46 empleados trabajan en la supervisión del servicio ${ }^{28}$ utilizando camionetas para el antigraffiti y la recolección de llantas tiradas en la calle.

La concesión de la gestión integral de los residuos sólidos ha ofrecido la posibilidad de incursionar en proyectos alternos o nuevos proyectos ambientales como el reciclado, la generación de energías renovables o el aprovechamiento de las llantas, entre otros. ${ }^{29}$ En este sentido, el gobierno ha dado un nuevo giro económico para que algunas empresas con capacidad de inversión, experiencia y conocimiento brinden a la ciudadanía oportunidades de empleo en un rubro que antes era totalmente desconocido: la externalización de un servicio público.

La certificación recientemente obtenida por parte de la PROFEPA como industria limpia en las dos áreas del servicio público, otorga mayor confiabilidad a la iniciativa privada para otro tipo de proyectos. En este sentido, PASA tiene la expectativa de renovar la concesión por un periodo igual de tiempo. 


\section{EL CONTROL GUBERNAMENTAL SOBRE EL CONCESIONARIO}

La relación entre PASA y la Dirección de Limpia es muy directa, con la mejor disponibilidad para el diálogo en caso de alguna discrepancia o alguna situación extraordinaria. Aunque esta relación tuvo un proceso de ajuste al inicio de la concesión, se acordó trabajar de acuerdo a las experiencias de la empresa implementando las prácticas exitosas de recolección de otras ciudades.

La colaboración es muy estrecha, ya que se pide apoyo a la empresa para eventos especiales como desfiles o para las visitas de funcionarios o del ejecutivo de otros niveles de gobierno, entre otros. Dada la calidad del servicio que presta PASA, los cambios en la administración municipal no le han afectado.

El aprendizaje del municipio con esta concesión es que se puede impulsar un modelo de servicio de recolección de basura basado en la planeación estratégica.

Se ha aprendido que es posible hacer cambios positivos en la cultura de la disposición y recolección de basura; por ejemplo se han implementado técnicas de movilidad del personal para evitar el pago de propinas, evitar la pepena y se ha modificado el uso de los tambos de 200 litros como contenedores de almacenaje de basura. ${ }^{30}$

La gestión de los residuos por parte de la iniciativa privada permite a la Dirección de Limpia enfocarse en la planeación a corto, mediano y largo plazo, registrando los nuevos fraccionamientos para identificar las futuras rutas a partir de su habilitación, es decir, se puede medir con antelación el aumento del servicio donde corresponda para cubrir la demanda de forma inmediata.

La evaluación anual de la gestión de residuos se realiza de manera conjunta entre la Dirección de Limpia y la empresa PASA con el fin de intercambiar información general - 
referente a nuevas solicitudes, nuevas rutas, personal, vehículos y supervisión necesaria, etc.- e implementar mejoras en el operativo.

\section{CONCLUSIONES}

A cinco años de la gestión integral de los residuos urbanos en Ciudad Juárez, a partir de la modalidad de concesión a la iniciativa privada, existe un aprendizaje tanto para el gobierno municipal como para la empresa prestadora del servicio. Por un lado, el gobierno municipal ha asimilado la necesidad de la planeación como parte imprescindible de su trabajo, se ha instruido con nuevas propuestas para realizar los operativos con mayor eficiencia y ha aprendido sobre la necesidad de constituir grupos especializados de trabajo para generar nuevos programas destinados al mejoramiento de la gestión integral de residuos. Por otra parte, la empresa reconoce que, a mayor eficiencia y calidad del servicio, mayores serán sus posibilidades de renovar la concesión y extender los servicios para eventos especiales y el manejo de otros rubros de limpia, lo cual le generará mayores ganancias económicas.

En este aprendizaje destacan algunas prácticas innovadoras en la gestión del servicio:

- La concesión del servicio permite al personal del municipio destinar mayores espacios y recursos humanos a la planeación estratégica, pues anteriormente, por el manejo y control del operativo completo, no se disponía del tiempo suficiente para esta actividad.

- El municipio puede llevar a cabo un rol articulador y de control con empresas privadas que brindan servicios de calidad a la población bajo una estricta supervisión de las mismas; en este caso se realiza una doble supervisión del servicio, por parte de la empresa y del municipio.

- El sistema de atención a las quejas del servicio de recolección de animales muertos o de atención a tiraderos clandestinos, entre otros, se ha reforzado y agilizado a partir de la 
supervisión conjunta, de la fijación de vehículos para las rutas con radios de recolección y del monitoreo coordinado de la empresa y el municipio.

- Las pautas culturales de la disposición de basura por los particulares se han modificado al sustituir el uso del tambo de 200 litros por bolsas o contenedores de menor capacidad; el aumento de la frecuencia de recolección facilita el operativo, que requiere de menor tiempo para cumplirse.

- Se ha erradicado la cultura de pago o compensación económica «simbólica»—esto es, el pago de menos de 10 pesos por recolectar la basura domiciliaria - a los empleados del servicio de limpia, ya que el municipio cubre el pago total del servicio. Al mismo tiempo, se ha trabajado en la supresión de la pepena por medio de las supervisiones conjuntas entre la empresa y el municipio.

- La implementación del uso de uniformes de los operadores, la identificación de las unidades de recolección y el estricto cumplimiento de los días y horarios de servicio, han permitido optimizar la cobertura de la recolección domiciliaria a pesar del cierre u obstrucción a la circulación vehicular que se ha implementado en algunas colonias debido a la inseguridad.

- La colaboración del sector privado con el gobierno en la prestación de un servicio público es una práctica novedosa y exitosa en la ciudad y ha aumentado el interés por extenderlo a otros servicios.

- La normatividad en cuestión de residuos sólidos en el estado se ha promovido para ofrecer garantías a los particulares, apoyadas en las legislaciones de otros ámbitos del gobierno. 


\section{BIBLIOGRAFÍA}

Aguilar Villanueva, Luis F., 2006, Gobernanza y gestión pública, Fondo de Cultura Económica: México.

Código municipal para el estado de Chihuahua, 2004a, División de Documentación y Biblioteca, Chihuahua.

Congreso del Estado, 2011, Ley de Desarrollo Urbano del Estado de Chihuahua, Última Reforma POE 2007.01.13/No.4, Secretaría de Servicios Jurídico Legislativos, División de Documentación y Biblioteca, Chihuahua.

Comisión de Cooperación Ecológica Fronteriza (COCEF), 1998, en Noticocef, enero, en http://www.cocef.org/apartcom/en98.htm [consulta: febrero de 2008].

Constitución Política de los Estados Unidos Mexicanos (CPEUM), 2005, última reforma publicada en el Diario Oficial de la Federación, 20 de junio.

Córdova Bojórquez, Gustavo, Lourdes Romo Aguilar, Cecilia Sarabia Ríos e Israel Díaz Arcos, 2006, «Los actores y la privatización del servicio de limpia en Ciudad Juárez», en Estudios Fronterizos, julio-diciembre, v. 7, n. 14, UABC, Mexicali, pp. 113-148.

Del Pino, Eloísa, 2004, Los ciudadanos y el Estado: las actitudes de los españoles hacia las administraciones y las políticas públicas, INAP, Madrid.

Dirección General de Ecología y Protección Civil, Gobierno Municipal 2010-2013, 2011a, Residuos recolectados y dispuestos en el 2010 y 2011, mimeo.

— $2011 b, \quad$ Relación de empresas y/o personas autorizadas por la Dirección de 
Normativa Ambiental para la recolección de residuos sólido son peligrosos, en http://juarez.gob.mx/clima/rsnp.pdf

Fuentes, Cesar, 2009, «La planeación estratégica como instrumento de un nuevo modelo de gestión del desarrollo urbano en Ciudad Juárez: retos y oportunidades», en La gestión del desarrollo local en México: problemas y agenda, coordinado por Ramos, José María e Ismael Aguilar Barajas, El Colegio de la Frontera Norte, Porrúa, México.

García del Castillo, Rodolfo, 1998, «Administración del desarrollo urbano y servicios públicos municipales. Un marco de estudio», en Anaya Cadena, Vicente, Administración Pública, Centro de Estudios del Desarrollo Regional, Estatal y Municipal, Gobierno del Estado de Tlaxcala, México.

Hernández-Mendible, Víctor R., 1999, Concesión de servicios públicos y concesión de obras públicas. Servicios públicos. Balance \& perspectivas, Vadell Hermanos Editores, Caracas.

Instituto Nacional de Estadística, Geografía e Informática, 1986, Estadística de la industria maquiladora de exportación 1975-1985, INEGI, México.

Instituto Nacional de Estadística y Geografía, 2010, Chihuahua, compendio censal del siglo $X X$, INEGI, México.

Ley General para la Prevención y Gestión Integral de los Residuos (LGPGIRS), 2003.

Milward, H. Brinton, Keith G. Provan y Barbara A. Else, 1998, ¿Qué es el "Estado hueco?, en La gestión pública. Su situación actual, coordinado por Bozeman, Barry, Fondo de Cultura Económica, México, pp. 411-428. 
Periódico El Norte, 2011, Limpiando Juárez, 19 de julio, GEN-PASA, Ciudad Juárez.

Ramió, Carles, 2002, Teoría de la organización y administración pública, Tecnos, Universitat Pompeu Fabra, España.

Reglamento Orgánico de la Administración Pública del Municipio de Juárez, Estado de Chihuahua, 2011, aprobado en sesión n. 11 del 23 de diciembre de 2004, publicado en el Periódico n. 12 de 9 de febrero de 2005.

Rubio Salas, Rodolfo, 2011, «La dinámica demográfica del municipio de Juárez en la primera década del siglo XXI», en Foro bienal de estudios del desarrollo. Los nuevos enfoques del desarrollo, (memoria electrónica), coordinado por Gutiérrez Casas, Luís Enrique, Red Iberoamericana de Estudios del Desarrollo y Universidad Autónoma de Ciudad Juárez

Santiago Sánchez, Federico Fabricio, 2009, «Elementos para delimitar los servicios públicos en México», en Actualidad de los servicios públicos en Iberoamérica, coordinado por Cienfuegos Salgado, David y Luís Gerardo Rodríguez Lozano, Instituto de Investigaciones Jurídicas, UNAM, México, pp. 645-680.

Santos Zavala, José (2006), «Gestión integral de residuos sólidos. El caso del programa "Basura que no es basura” León Guanajuato» en Gobierno de proximidad la capacidad y el ingenio de la gestión local mexicana coordinado por Tonatiuh Guillén, Liliana González Pantoja y Pablo Rojo Calzada, CIDE, SEGOB, COLEF, México, pp. 165-172.

Sarabia, Cecilia, 2011, entrevista a Martín Moreno Moreno, gerente de servicios en Ciudad Juárez de Promotora Ambiental de la Laguna, 12 de julio, La gestión integral de los residuos sólidos urbanos en Juárez: lecciones innovadoras para otros municipios. 
Sarabia, Cecilia, 2011, entrevista al Lic. Jorge Alberto Gutiérrez Casas, director de servicios públicos municipales 2007-2013, 14 de julio, La gestión integral de los residuos sólidos urbanos en Juárez: lecciones innovadoras para otros municipios.

Secretaría del Ayuntamiento de Ciudad Juárez, 2006, Concesiones otorgadas por el municipio de Ciudad Juárez, en: http://www.juarez.gob.mx/transparencia/Documtos/Concesion\%20limpia.pdf [consulta: julio 2011].

Universidad Autónoma de Ciudad Juárez, 2011, Estudio de percepción ciudadana con una georeferenciación de los datos en los diversos sectores que abarca el operativo PASA, abril, UACJ, Chihuahua.

\section{NOTAS}

${ }^{1}$ Los datos completos de población y tasa de crecimiento por año son: 1950: 131,308 hab.; 1960: 276,995 hab.; 1970: 424,135 hab.; 1980: 567,365; 1990: 798,499 hab.; 2000: 1,218,817 hab.; y 2010: 1,332,131 hab.

${ }^{2}$ La Ley General para la Prevención y Gestión Integral de los Residuos define residuos de manejo especial como aquéllos generados en los procesos productivos que no reúnen las características para ser considerados como peligrosos o como residuos sólidos urbanos, o que son producidos por grandes generadores de residuos sólidos urbanos.

${ }^{3}$ Del total del padrón, corresponden por tipo de autorización: residuos especiales 3, escombro 8 , residuos de aceite 5, trampas de grasa 9, residuos alimenticios 13, basura industrial 22, residuos sólidos reciclables 41, basura 10 .

${ }^{4}$ Los artículos 118, 131 y 2007 establecen a la figura física o moral como responsable de las acciones urbanas por parte del ayuntamiento y detallan el tipo de sanciones por infracciones a este precepto que van desde la amonestación, la multa o la demolición hasta la suspensión de las obras, instalaciones o servicios.

${ }^{5}$ Un ejemplo claro de esta práctica existe desde el año 1976, cuando la administración prí́sta, con la oposición del PAN, intentó concesionar el servicio de limpia (Córdova, et al. 2006: 128). Veinte años después, y durante la administración del alcalde panista Ramón Galindo, el asunto se reinvirtió. El Sindicato de Trabajadores del Municipio y los dirigentes del PRI local manifestaron un rotundo no al plan piloto de concesión del servicio de limpia.

${ }^{6}$ El término burocracia se aplica, de acuerdo con la concepción de Weber, como un cuerpo organizado para alcanzar los fines del Estado.

${ }^{7}$ Este autor señala que existen dos tendencias en la administración pública contemporánea: la primera se orienta a reivindicar, recuperar y reconstruir su naturaleza pública, y es el resultado del despertar de los ciudadanos que «se pronuncian frente a los asuntos públicos que son de interés para la sobrevivencia, convivencia y calidad de vida personal y social y que luchan por determinar los temas de la agenda de gobierno y la política pública»; la segunda busca recuperar, reactivar y reconstruir la capacidad administrativa 
y «guarda indudablemente relación directa con las medidas de ajuste y equilibrio hacendario» (Aguilar 2006: 41).

${ }^{8}$ La concesión de servicios por parte del Gobierno federal funciona en el servicio de Seguridad Social.

9 Ramió (2002) menciona que la producción de servicios por parte de la administración pública bajo determinados estándares de calidad se denomina servucción, y debe contar con soporte físico y humano para la prestación del servicio, un sistema de organización interno, clientes o beneficiarios del servicio y las relaciones que se establecen entre estos elementos (ciudadano cliente-prestador del servicio; parte visible de la organización-parte invisible de la organización; relaciones de concomitancia entre ciudadanos cliente).

${ }^{10}$ El caso del servicio de limpia se especifica en el art. 115, fracción II, inciso c, donde se establece que los municipios tendrán a su cargo las funciones y servicios públicos de limpia, recolección, traslado, tratamiento y disposición final de residuos.

${ }^{11}$ En el caso del servicio de limpia se identifica la externalización «porque está establecido dentro de la Constitución [de los Estados Unidos Mexicanos], en el art. 115 [se establece que] es una responsabilidad de la administración municipal el servicio público, no lo podemos privatizar, [no se puede privatizar,] no vendimos nada, simple y sencillamente la responsabilidad y la facultad sigue siendo totalmente de la administración [el manejo de eso.] ¿Qué hacemos?, pues contratamos el servicio pero [lo seguimos, y] seguimos siendo los responsables ante la ciudadanía del mismo» (Jorge Alberto Gutiérrez Casas, director de Servicios Públicos Municipales 2010-2013).

${ }^{12}$ Reglamento Orgánico de la Administración Pública del Municipio de Juárez, arts. 46 y 49.

${ }_{13}$ Datos proporcionados por el Lic. Jorge Alberto Gutiérrez Casas, director de Servicios Públicos del municipio 2010-2013.

${ }^{14}$ En este sentido, el Lic. Jorge Alberto Gutiérrez Casas comenta que «existía un problema de pepenadores, pues [el alto número de éstos.] En ese tiempo teníamos un registro de aproximadamente 120 [pepenadores,] y llegaba a haber hasta más de 480 [pepenadores tanto en la celda, pues] era ya toda una población que hacía muy ineficiente el manejo de las maquinas ahí, inclusive se presentaron incidentes [hasta] fatales [,verdad,] por la voracidad de esta gente $[\mathrm{de} \ldots$ de...] de atravesarse en los camiones que terminaban atropellados o enterrados en la misma basura».

${ }^{15}$ Lic. Jorge Alberto Gutiérrez Casas, director de Servicios Públicos del municipio 2010-2013, señala: «Nosotros estamos totalmente convencidos que para tener un gobierno eficiente [necesitamos, o sea,] no se trata de crear una estructura [en...] pesada, una estructura pulpo y mucho menos tratar de involucrarse en aquellos proyectos que también traen beneficio económico porque [no es parte de] la función del gobierno es dar el servicio, el mejor servicio, la mayor calidad, garantizar esa calidad de vida del ciudadano, eso es un hecho».

${ }_{16}$ «Artículo 49.- El Director de Limpia tendrá a su cargo la recolección de basura en toda la ciudad y su traslado y disposición final en el relleno sanitario, de conformidad a la normatividad aplicable. En caso de que el servicio de recolección de basura se concesione, quedará a cargo de la recolección de basura en los edificios públicos municipales, y será responsable de supervisar y verificar que la empresa concesionaria cumpla con el traslado y disposición final de los residuos sólidos en estricto apego a los procedimientos establecidos en el contrato de concesión y de igual forma vigilará que ésta, cumpla con la normatividad vigente en la materia» (Reforma publicada en el Periódico Oficial del Estado, n. 71, el 4 de septiembre de 2010).

${ }^{17}$ Según se informa en la sección de transparencia del municipio, en www.juarez.gob.mx.

${ }^{18}$ El mantenimiento consiste en cambio de aceite, filtro, revisión de balatas, de luces, de frenos, etcétera.

${ }^{19}$ Martín Moreno, gerente de servicios de PASA, comenta al respecto que «[lo que se da básicamente,] no generan mucha basura [, entonces] por eso se dividió [en...] en esas formas. Lo que es acá para ANAPRA, [este...] para toda la parte de arriba, también vamos tres veces por semana y aun así con la terracería como está, [bueno pues,] nos hemos ido adecuando a conocer las rutas [y...] y hablando con la gente, [verdad,] para poder tener mayor servicio».

${ }^{20}$ El motivo de las quejas se debe a que algún domicilio no recibió el servicio. 
${ }^{21}$ Entre los programas impulsados por la Dirección de Limpia se encuentra el de Destilichadero, programa de carácter permanente que recolecta toda la basura pesada y/o voluminosa. Por su parte, la administración 20072010 del municipio de Juárez menciona en marzo de 2008 la creación de un nuevo programa de educación ambiental llamado Guardianes del medio ambiente, el cual funciona a base de folletos, panfletos, trípticos, visitas a los centros educativos desde niveles básicos a universitarios, asociaciones civiles, maquiladoras, etc. En abril de 2008, el programa citado comenzó a operar con un módulo en la plaza principal con motivo de la "feria ecológica" organizada por la Dirección de Ecología y Protección Civil en conjunto con la Dirección General de Servicios Públicos Municipales. En dicho módulo, se explicaba a los asistentes el proceso de aprovechamiento de la basura por la extracción de biogás y generación de energía eléctrica, y también se impartieron pláticas a niños y niñas sobre calentamiento global, preservación del ambiente, y cómo elaborar manualidades con productos reciclables (Chihuahua al instante, 7 de abril de 2008).

${ }^{22}$ La Ley General para la Prevención y Gestión Integral de los Residuos (LGPGIRS) define gestión integral de residuos como: «Conjunto articulado e interrelacionado de acciones normativas, operativas, financieras, de planeación, administrativas, sociales, educativas, de monitoreo, supervisión y evaluación, para el manejo de residuos, desde su generación hasta la disposición final, a fin de lograr beneficios ambientales, la optimización económica de su manejo y su aceptación social, respondiendo a las necesidades y circunstancias de cada localidad o región» (LGPGIRS, 2003).

${ }^{23}$ Gobierno municipal de Juárez. Segundo informe de gobierno 2004-2007.

${ }^{24} \mathrm{La}$ evaluación tuvo como objetivo la medición representativa de la percepción del servicio, que consideró como universo de estudio el número de viviendas en Ciudad Juárez de acuerdo al Conteo 2005 del INEGI, obteniendo una muestra de 1,585 viviendas como objeto de estudio en 187 rutas. Para el análisis de la información, se combinó el análisis estadístico con el análisis geoespacial para dar representación visual a los datos, utilizando en este último método la moda como medida de tendencia central.

${ }^{25}$ Como una medida de los autores de corroboración de la información de la evaluación de la UACJ, el sondeo consistió en una encuesta enviada vía correo electrónico a usuarios con la intención de corroborar la información y obtener algunas opiniones sobre el servicio de limpia, cuya muestra abraca a usuarios de las dos zonas de operación de PASA.

${ }^{26}$ Norte de Ciudad Juárez. 29 de noviembre de 2011. ¡Felicidades Ciudad Juárez, Chihuahua! Por ser la ciudad más limpia de México, y por ello triunfadora en el concurso Limpiemos Nuestro México, 8B.

${ }^{27}$ Tradicionalmente el camión recolector de basura anunciaba su llegada al sector o calle mediante el uso de una campana, cultura que ha erradicado el concesionario.

${ }^{28}$ Antes de la concesión, el Departamento de Limpia operaba con 750 empleados, más un número no preciso de supervisores.

${ }^{29}$ El Lic. Jorge Alberto Gutiérrez Casas, director de Servicios Públicos Municipales, comenta al respecto: «ahorita tenemos ya una planta generadora de energía eléctrica a través del biogás, de los gases emanados de la descomposición de la basura, tenemos una inversión de más de cuatro millones de dólares en una planta recicladora [que...] seleccionadora que ya empieza a operar y que nos permitió darle un empleo digno a los pepenadores; hoy día el relleno sanitario no cuenta con pepenadores, no hay un solo pepenador en la zona, [en la...] ahora hay empleados de una planta recicladora instalada en el relleno sanitario, [sí,] que le da empleo aproximadamente a 250 trabajadores, anteriormente pepenadores, o sea [no,] vimos solución a un problema social que se vivía en esa área; tenemos dos trituradoras de llantas que nos ha permitido que junto también con [un...] la planta cementera, entre las tres nos han permitido que en los últimos cinco años haya una reducción de más del 50\% [del...] del centro de acopio de llantas; anteriormente se contemplaba que había casi seis millones de llantas en el centro de acopio, hoy día lo puedes [ya ver y tú] constatar, hay menos de tres millones de llantas [ya] ahí, [¿por qué?] Porque existen dos plantas que trituran llanta y [que trituran he... la llanta y] le sacan el caucho, el acero, y lo [y uno lo] exportan y otro lo comercializan aquí [para...] para combustible, sin contaminación, a la planta cementera».

${ }^{30}$ La importancia de erradicar esta cultura de utilización de tambos radica en que la acumulación de basura en este tipo de contenedores permitía la proliferación de mosquitos en verano, además de que se podía prender fuego a la basura y esto se convertía en otro foco de contaminación al medio ambiente. 
Fecha de recepción: 25 de julio de 2011.

Fecha de aceptación: 5 de diciembre de 2011. 